\section{Suicidio en poblaciones lesbiana, gay, bisexual y trans: revisión sistemática de una década de investigación (2004-2014)}

\author{
ALEMKA TOMICIC ${ }^{1,2, a}$, CONSTANZA GÁLVEZ $Z^{1, c}$, \\ CONSTANZA QUIROZ ${ }^{1, c}$, CLAUDIO MARTÍNEZ $Z^{1,2, a}$, \\ JAIME FONTBONA ${ }^{3, b}$, JULIANA RODRÍGUEZ ${ }^{4, b}$, \\ FRANCISCO AGUAYO ${ }^{5, b}$, CATALINA ROSENBAUM ${ }^{1, c}$, \\ FANNY LEYTON ${ }^{6, d}$, ISIDE LAGAZZI ${ }^{7, c}$
}

\section{Suicide in lesbian, gay, bisexual and trans populations: systematic review of a decade of research (2004-2014)}

Background: Lesbian, gay, bisexual and trans (LGBT) populations have a high prevalence of suicide behaviors. Aim: To review the literature on suicide among LGBT populations. Material and Methods: A systematic review of the scientific literature published between 2004 and 2014 on suicidality among LGBT people was conducted. Forty-five articles were selected for a thematic analysis. Results: The research on suicide among LGBT people has increased during the last decade. The vast majority of studies have been conducted in the U.S. and other English speaking countries. At the same time, in a great part, these studies have been published in journals specializing in mental health and public health of sexual minorities. In general, they provide a significant foundation on protective and risk factors for suicide as well as their epidemiology. Conclusions: The focus of research on suicide among LGBT populations is mainly exploratory. More attention on these at-risk groups is required from the suicide research community in order to provide useful evidence for prevention and development of focalized and effective interventions.

(Rev Med Chile 2016; 144: 723-733)

Key words: Gender Identity, Mental Health, Suicide, LGBT.

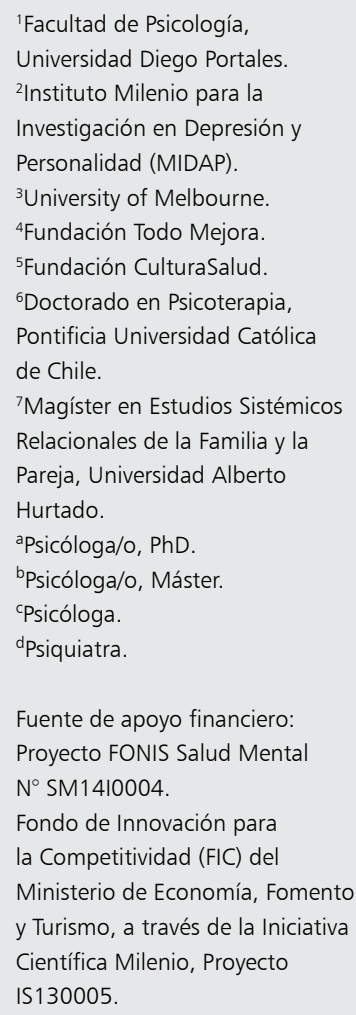

Recibido el 10 de agosto de 2015, aceptado el 12 de enero de 2016.

Correspondencia a: Alemka Tomicic Facultad de Psicología, Universidad Diego Portales Grajales 1898, Santiago. Teléfono: + 56977096048 alemka.tomicic@udp.cl
$\mathrm{E}$ n el año 2003 el suicidio fue declarado como un problema de salud pública por la Organización Mundial de la Salud, posicionándolo en el $8^{\circ}$ lugar entre las enfermedades con más años de vida perdidos al 2003 y en el lugar $14^{\circ}$ en estimación de carga de enfermedad y años de vida perdidos por discapacidad al año 2002 ${ }^{1}$. Entre los grupos de riesgo de comportamiento suicida, las poblaciones de personas lesbianas, gays, bisexuales $y$ trans (LGBT) han sido las que han mostrado una de las más elevadas prevalencias en este problema de salud mental ${ }^{2-6}$.

La asociación entre población LGBT y riesgo suicida ha sido entendida desde el enfoque de los determinantes sociales de la salud ${ }^{7}$. Al respecto, se señala que la población LGBT se ve altamente afectada por problemas de salud mental relacionados con el estigma y la discriminación ${ }^{5,8}$. Específicamente, el modelo de stress en minorías ${ }^{8}$ ha ofrecido una manera de comprender cómo la 
pertenencia a una minoría discriminada, en este caso ser parte de la población LGBT, deja expuesto al individuo a un entorno social hostil caracterizado por el prejuicio, el rechazo y la exclusión. Este entorno generaría problemas de salud mental tales como depresión, abuso de sustancias, aislamiento social, conflicto con los pares y victimización, lo cual incrementaría los factores de riesgo individuales para el suicidio ${ }^{4,8,9}$.

La consideración de la orientación sexual y la identidad de género como un determinante de la salud en términos generales y, específicamente, como una condición de riesgo para la ideación e intento suicida ${ }^{4}$, obliga el desarrollo de un conocimiento culturalmente sensible sobre esta problemática de salud. Haas et al. (2010) recomiendan promover la inclusión de preguntas sobre orientación sexual e identidad de género en la investigación sobre suicidio y riesgo suicida, así como también la implementación de estudios para determinar qué aspectos de la vida de las personas LGBT se relacionan más fuertemente con problemáticas de salud mental. Asimismo, plantean la necesidad de hacer visibles las necesidades de salud mental de las personas LGBT para promover el diseño de intervenciones específicas y pertinentes ${ }^{7}$.

En Chile y Latinoamérica sólo unos pocos estu$\operatorname{dios}^{10-14}$ han focalizado en las condiciones de salud mental y sus determinantes sociales en personas sexualmente diversas. Es evidente, al menos en lo que concierne a la actividad científica, que los problemas de salud mental y de suicidabilidad no han formado parte de la agenda investigativa de la región y, por lo tanto, no se cuenta con un conocimiento especializado sobre esta problemática ni con orientaciones específicas para su intervención, lo que habla a su vez de la invisibilidad de las necesidades de la población LGTB para las políticas y programas en salud pública.

En consideración con lo anteriormente planteado, realizamos una revisión de la literatura científica publicada a nivel mundial en el último decenio sobre suicidabilidad en población LGBT, con el propósito de proveer un cuadro comprensivo sobre la manera en que dicho conocimiento se ha generado, sobre sus principales hallazgos y conclusiones, y acerca de sus limitaciones a ser consideradas para el desarrollo futuro de esta línea de investigación en el contexto Latinoamericano.

\section{Material y Método}

Se realizó una revisión sistemática de la literatura entre los años 2004-2014 en revistas científicas indexadas de habla inglesa e hispana, con el propósito de analizar los estudios que hacen referencia directa o indirectamente a investigaciones en ideación suicida, intento suicida o suicidio consumado en población Lesbiana, Gay, Bisexual y Trans.

\section{Identificación de las publicaciones}

La búsqueda de los estudios publicados se realizó por medio de las siguientes bases de datos: MEDLINE (EBSCO host), Pro Quest Psychology Journals, Psyc INFO (EBSCO host) y Scielo. Los términos de búsqueda empleados fueron "suicide" $y$ "LGBT". Estos fueron utilizados de manera individual o de forma combinada con los siguientes términos: "suicide", "lesbian", "gay", "bisexual", "transexual".

El criterio temporal de búsqueda de los artículos consistió en que fueran publicados en los últimos diez años desde marzo de 2004 hasta agosto de 2014. Al finalizar este período de búsqueda, se encontraron 2.857 registros de artículos concordantes con las palabras o combinación de palabras descritas anteriormente.

\section{Selección de publicaciones para revisión}

Los artículos identificados fueron pre-analizados a nivel de título y abstract. Este tamizaje permitió seleccionar las publicaciones para la revisión considerando como criterios de inclusión que (a) estas refirieran a población lesbiana, gay, bisexual y/o trans, y (b) que dieran cuenta de algún aspecto del comportamiento suicida (ideación, intento o suicidio consumado) en esta población. Se consideró como criterio de exclusión que la publicación fuese una revisión sistemática o un metaanálisis. A su vez, se excluyeron aquellos artículos teóricos o conceptuales; artículos que presentaran diseños de intervención/investigación, pero no su aplicación y resultados; capítulos de libros; y reportajes periodísticos.

Del total de búsqueda, se seleccionaron 45 artículos (Figura 1 y Tabla 1). El procedimiento de selección siguió las orientaciones de la declaración PRISMA (2009) ${ }^{15}$. En la Figura 1 se presenta un resumen del proceso de inclusión y exclusión. 


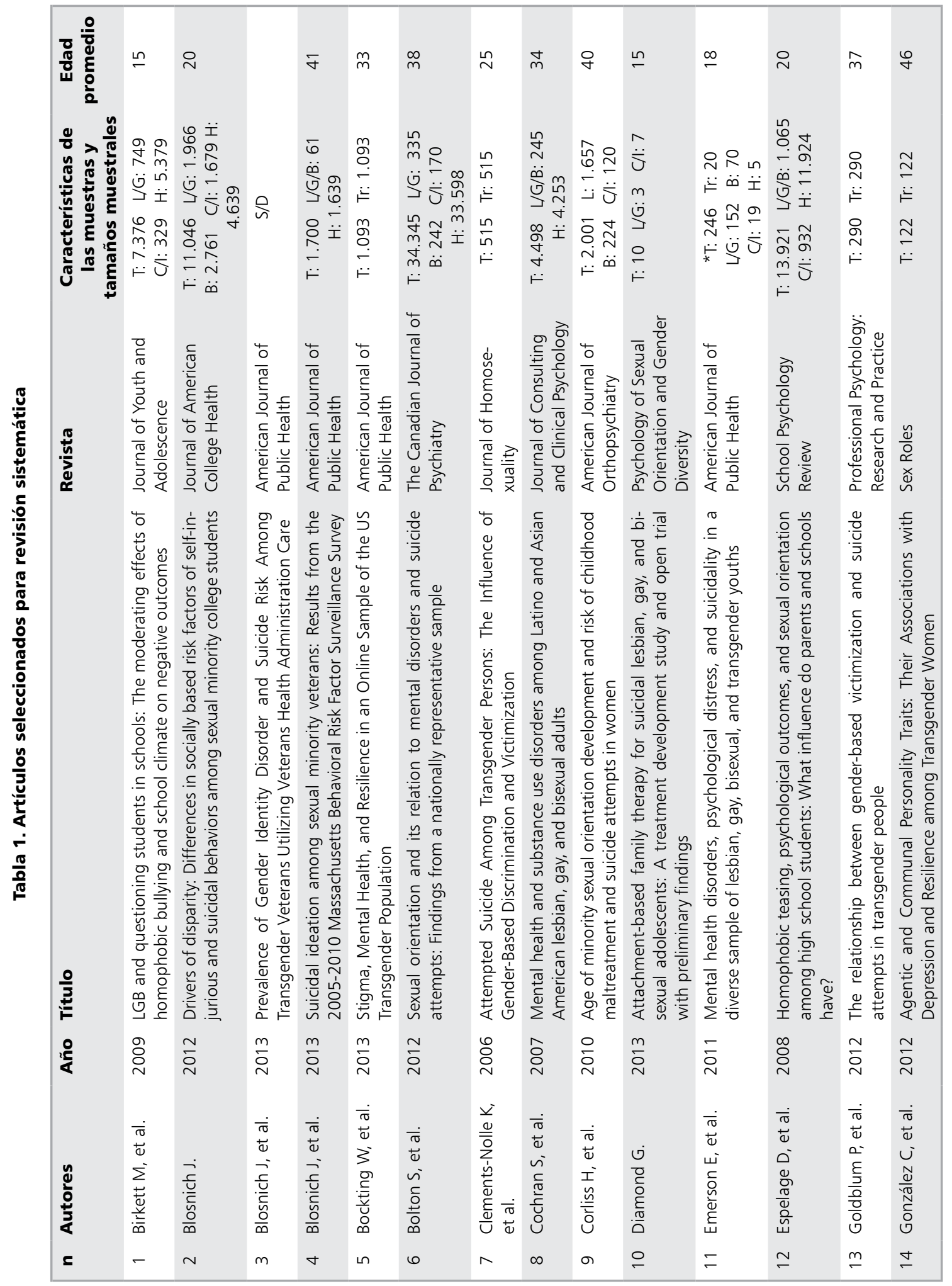




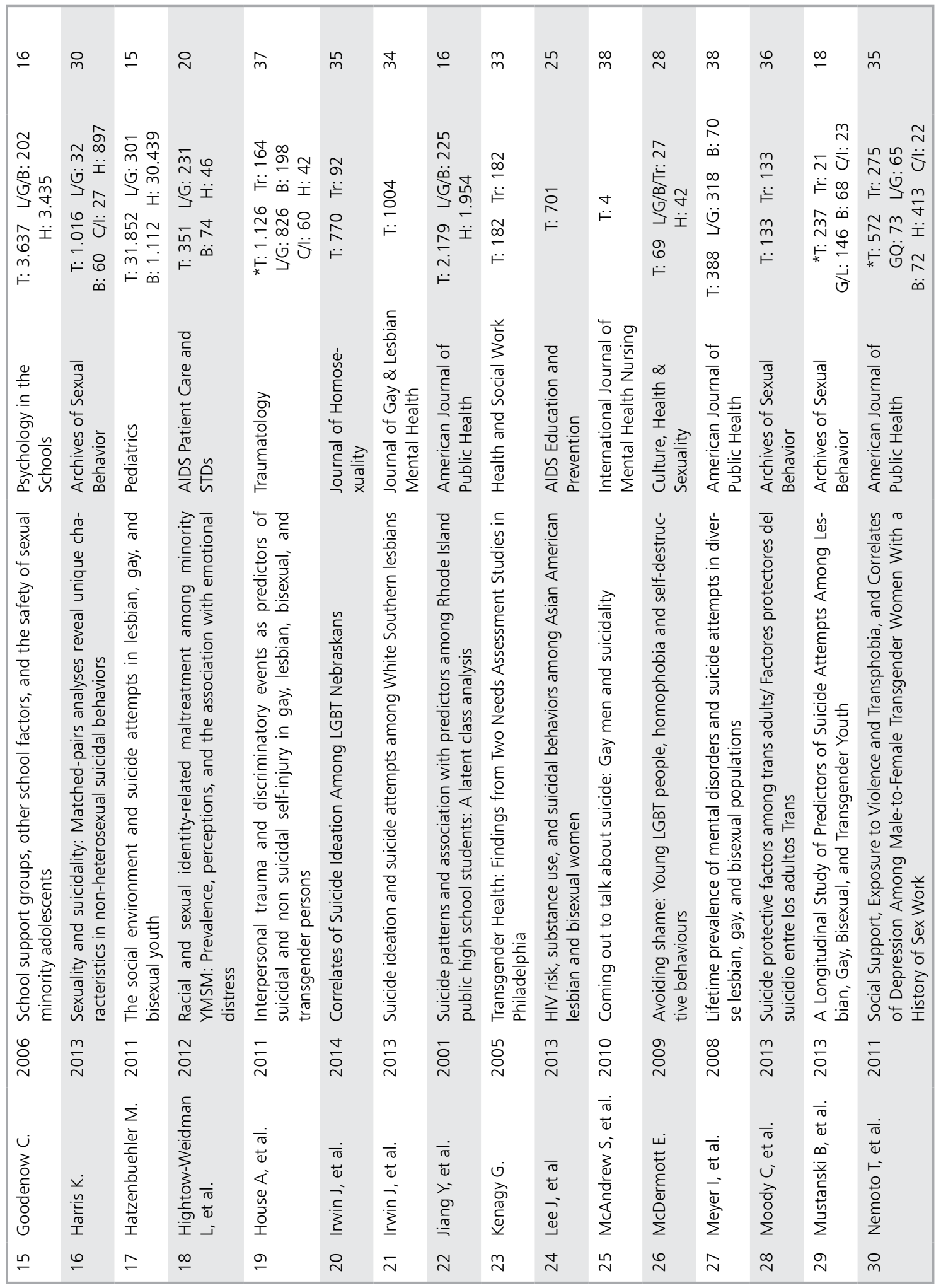




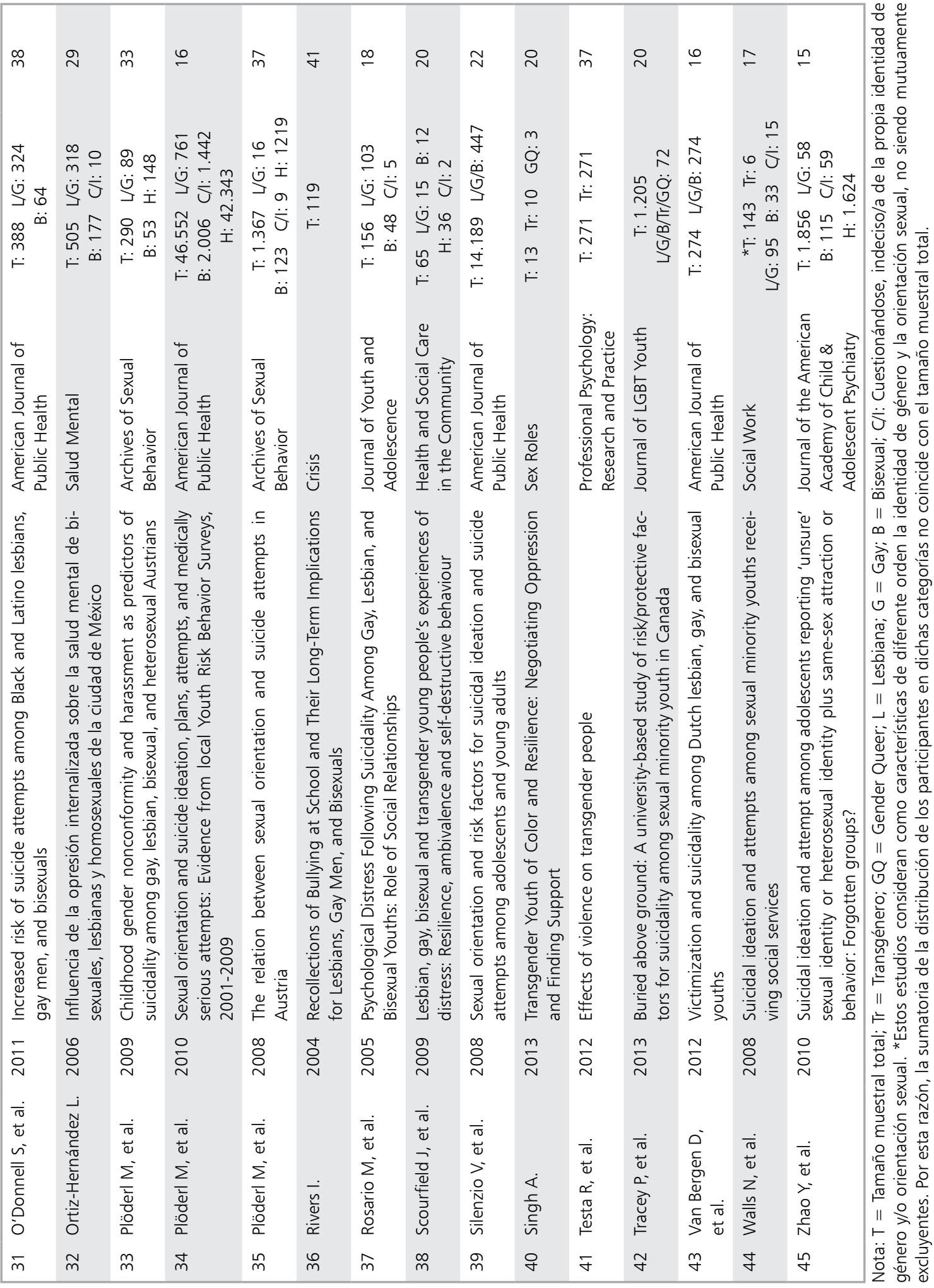




\section{Procedimiento de revisión}

Los artículos seleccionados fueron descargados $\mathrm{y}$ almacenados de manera digital (en formato PDF). Se construyó una base de datos para el

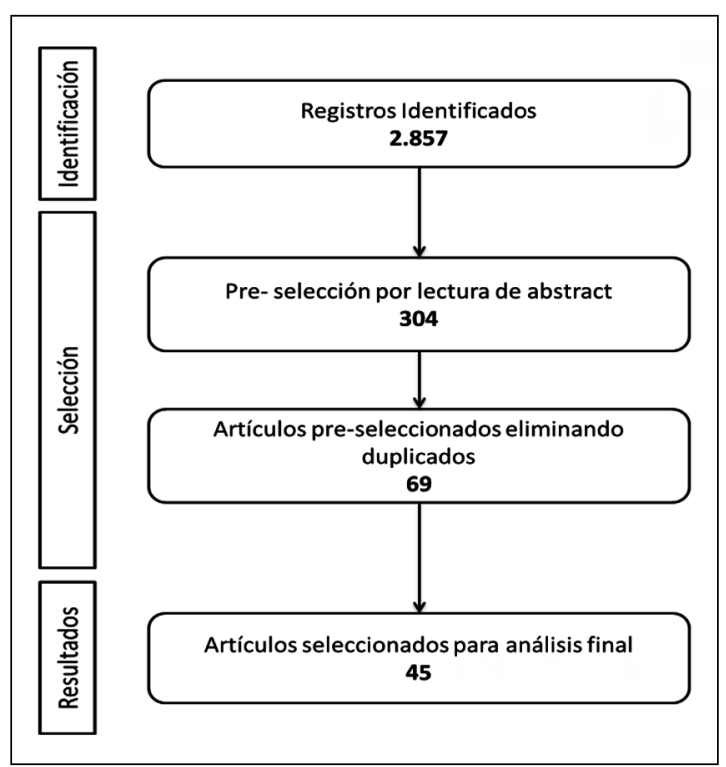

Figura 1. Diagrama de flujo que ilustra el proceso de inclusión y exclusión de los registros. análisis temático de cada uno de los artículos seleccionados, definiéndose 10 categorías de análisis (Tabla 2). Las categorías relativas a las condiciones de producción de las investigaciones fueron analizadas cuantitativamente, mientras que aquellas referidas a las bases conceptuales, y los principales resultados y conclusiones, fueron analizadas cualitativamente (i.e. síntesis temática) empleando el software Atlas.ti versión 7.

\section{Resultados}

Condiciones de producción de las investigaciones

En la década analizada, se observa un aumento sostenido de las publicaciones sobre suicidabilidad en población LGBT: 7\% en el bienio 2004-2005, 9\% en el bienio 2006-2007, 20\% en el bienio 20082009, 22\% en el bienio 2010-2011, y 42\% en el trienio 2012-2013-2014 (Figura 2). Por su parte, al desagregar por país, la mayor proporción de las investigaciones revisadas fueron realizadas en Norte América y lideradas por Estados Unidos de Norteamérica con 73\% (Canadá 7\%; México 2\%), mientras que $16 \%$ restante corresponde a estudios llevados a cabo en países europeos (Reino Unido 9\%; Holanda 2\%; Austria 2\%) y en Australia (2\%).

Con respecto a las revistas en las que estos

Tabla 2. Definición de categorías de análisis

\begin{tabular}{|c|c|}
\hline Nombre de la categoría & Definición \\
\hline Año & Año de publicación del artículo \\
\hline Revista & Revista en que fue publicado el artículo \\
\hline País & País en el cual el estudio fue realizado \\
\hline Conceptualización principal & $\begin{array}{l}\text { Corresponden a las principales conceptualizaciones sobre la identidad de género, la orien- } \\
\text { tación sexual, la relación entre población LGBT y salud mental, y sobre relación entre po- } \\
\text { blación LGBT y suicidio que se hallan a la base de la pregunta y/u objetivo de investigación }\end{array}$ \\
\hline Objetivo & $\begin{array}{l}\text { Corresponde a la delimitación del objeto de estudio explicitado en la investigación y que } \\
\text { señala la variable resultado del mismo, esto es la variable que constituye el foco del análisis }\end{array}$ \\
\hline Diseño & Enfoque metodológico cuantitativo, cualitativo o mixto \\
\hline Participantes & $\begin{array}{l}\text { Característica de la muestra en términos de la pertenencia a las categorías LGBT, y con } \\
\text { respecto al grupo etario }\end{array}$ \\
\hline $\begin{array}{l}\text { Instrumentos o dispositivos } \\
\text { de observación }\end{array}$ & $\begin{array}{l}\text { Técnicas e instrumentos de recolección de datos: Encuestas, técnicas cualitativas, autore- } \\
\text { porte u observación }\end{array}$ \\
\hline Resultados principales & $\begin{array}{l}\text { Corresponde a los principales resultados relativos al fenómeno del suicidio en la población } \\
\text { LGBT }\end{array}$ \\
\hline Alcances y Limitaciones & $\begin{array}{l}\text { Limitaciones y alcances de los resultados obtenidos en la investigación explícitamente } \\
\text { reportados por los autores }\end{array}$ \\
\hline
\end{tabular}




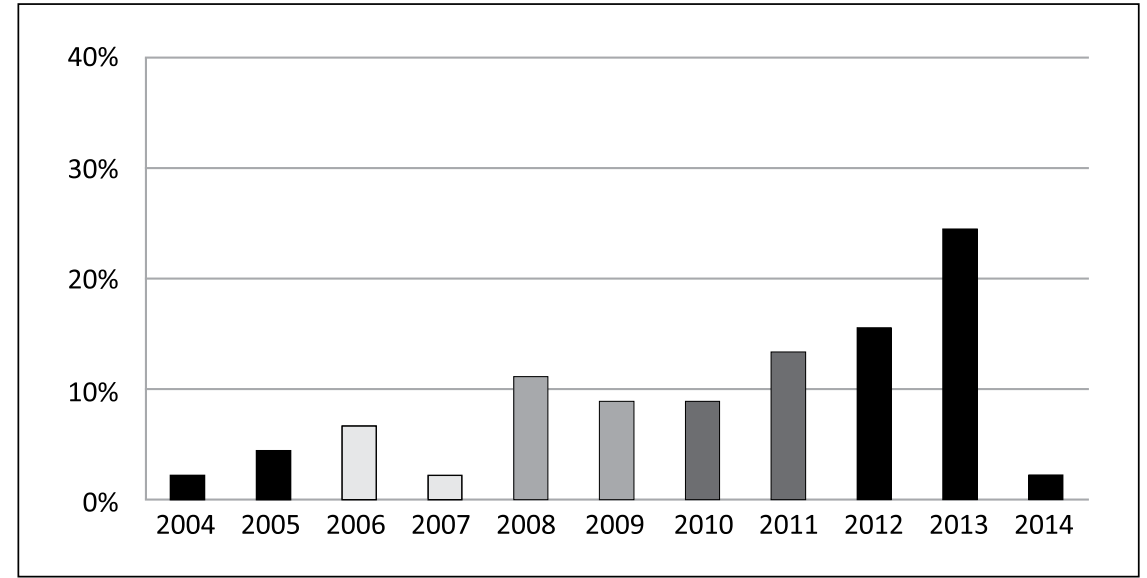

Figura 2. Distribución de publicaciones por año. estudios fueron publicados, llama la atención la variedad de las mismas, encontrándose que los 45 artículos analizados se distribuyeron en 27 revistas. El mayor porcentaje corresponde a la revista American Journal of Public Health con $24,4 \%$. Si se las analiza desagregándolas por área disciplinar o temática, más de la mitad de los artículos (60\%) fueron publicados en revistas del área de la salud y de la salud mental (e.g. American Journal of Public Health, International Journal of Mental Health Nursing, Journal of Consulting and Clinical Psychology, American Journal of Orthopsychiatry); $31 \%$ en revistas sobre sexualidad y género (e.g. Culture, Health \& Sexuality, Archives of Sexual Behavior); 9\% en revistas de psicología y trabajo social, y sólo un artículo en una revista especializada en suicido (The Journal of Crisis Intervention and Suicide Prevention). Asimismo, $16 \%$ de los estudios fueron publicados en revistas cuya población objetivo son niños, adolescentes y jóvenes (e.g Journal of the American Academy of Child \& Adolescent Psychiatry, Journal of Youth and Adolescence, Pediatrics).

El 50\% de las investigación tuvo por objetivo determinar factores de riesgo, protectores y/o de resiliencia en problemas de salud y salud mental en general, y de comportamiento suicida en particular, en personas lesbianas, gays, bisexuales y/o trans (e.g., Clements-Nolle et al., 2006; Munstanski et al., 2013), mientras que 24\% tuvo como propósito determinar la prevalencia e incidencia de ideación e intentos de suicidio en estas poblaciones (e.g. Plöderl et al., 2010). Un 13\% se orientó a la comprensión del proceso suicida en personas LGBT y a establecer la asociación entre dicho comportamiento y las experiencias de victimización de género, vale decir experiencias de discriminación y violencia debidas a su identidad de género y orientación sexual (e.g. Goldblum et al., 2012). Un 9\% buscó establecer la asociación entre pertenecer a alguna categoría de diversidad sexual y presentar sintomatología psiquiátrica (e.g. Emerson et al. 2011). Finalmente, sólo un estudio tuvo por propósito evaluar el impacto de una intervención para prevenir comportamientos suicidas en población LGBT (Diamond et al., 2013). Específicamente, en esta investigación se probó la efectividad de una terapia familiar para adolescentes lesbianas, gays y bisexuales basada en el apego, obteniéndose evidencia de altos niveles de adherencia al tratamiento y disminuciones significativas de ideación suicida, sintomatología depresiva, y estilos de relación asociados con apegos ansiosos y evitativos.

Considerando la pertenencia a las diferentes categorías de orientación sexual e identidad de género, $60 \%$ de los estudios tuvo por participantes a personas identificadas en alguna de las categorías de orientación sexual exclusivamente (LGB), 22\% tuvo participantes identificados como transgénero (T), y $18 \%$ restante incluyó ambos tipos de participantes (ver distribución desagregada en Figura 3). Con respecto al grupo etario de los participantes, cerca de la mitad de los estudios $(47 \%)$ consideró un rango amplio de adolescentes y adultos; la proporción restante focalizó en mayor medida en adolescentes y jóvenes (44\%) y, en menor medida, en adultos (9\%). 


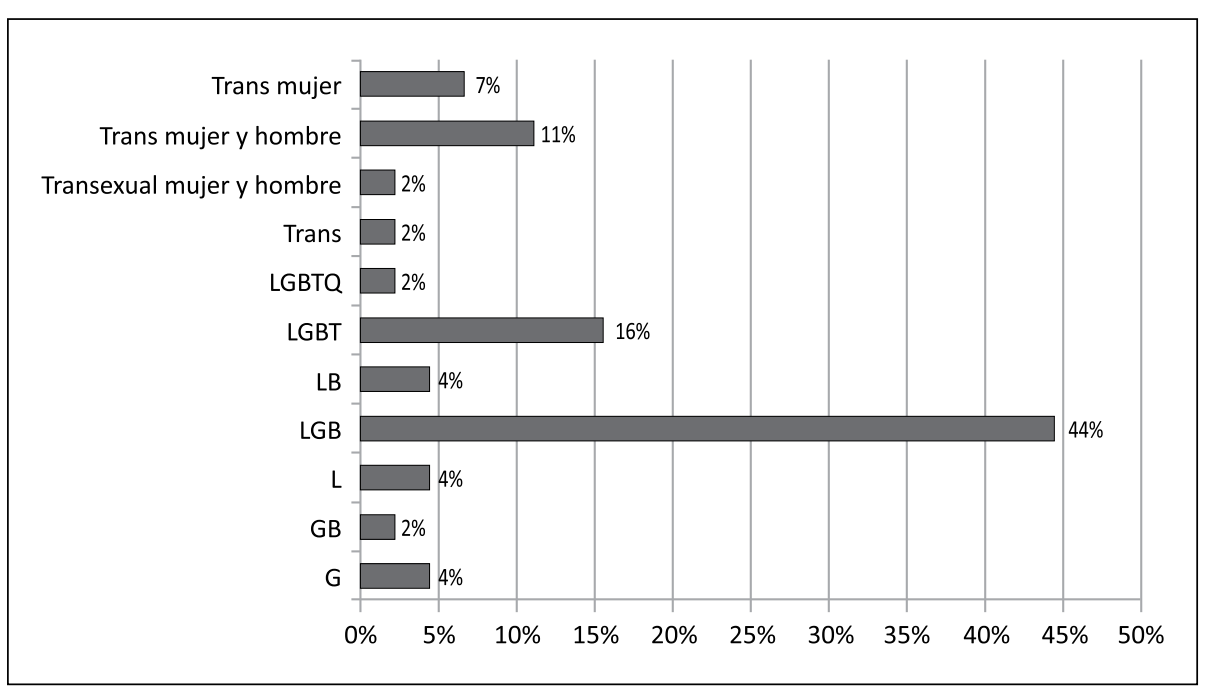

Figura 3. Distribución de publicaciones según categoría de orientación sexual e identidad de género de los participantes.
Del total de los artículos analizados, sólo tres (7\%) reportan diseños de investigación con metodologías cualitativas. En relación a las técnicas de recolección, la gran mayoría reporta el uso de técnicas de auto-reporte (44\% cuestionario electrónico y $44 \%$ cuestionario o entrevista estructurada) y sólo $12 \%$ reporta el uso de datos secundarios (e.g. Blosnich et al., 2013).

\section{Bases conceptuales de las investigaciones}

Respecto a la noción de orientación sexual existe consenso en la literatura revisada que esta corresponde a un fenómeno multidimensional, y que no hace referencia exclusiva a la conducta sexual sino que también a la identidad de género. En relación con esta última, se focaliza en la idea de la construcción de una identidad sexual diversa, siendo relevante la valoración de esta identidad en un continuo dimensional "vergüenza-orgullo" (McDermott et al., 2009).

Todas las investigaciones revisadas presentan el antecedente de que la población LGBT muestra peores indicadores de salud mental (i.e. sintomatología depresiva y ansiosa, abuso de sustancias y conductas autolesivas) cuando se la compara con población heterosexual, y que estos indicadores constituyen, a su vez, factores de riesgo para el suicidio. Estos problemas de salud mental no serían inherentes a la orientación sexual e identidad de género sino que se explicarían por determinantes sociales de la salud (i.e. modelo de estrés de minorías discriminadas; e.g. Bocktinget al., 2013; Plöderl et al., 2008).

Por otra parte, los estudios revisados coinciden en señalar que la suicidabilidad es un problema mayor en la población LGBT, presentando elevados índices de ideación e intento suicida. En conjunto, los estudios refieren en sus antecedentes tasas de intento de suicidio de entre 20 a 53\% para jóvenes LGB (e.g. O’Donnell et al., 2011) y de entre 23 a $47 \%$ para el caso de jóvenes trans (e.g. Testa et al., 2012). En términos comparativos, se señala que la probabilidad de ideación e intentos de suicidio es de hasta siete veces mayor entre jóvenes LGBT que entre sus contrapartes heterosexuales (e.g. Diamond, 2013).

Finalmente, respecto al suicidio consumado, Irwin et al. (2013) presentan la estimación de que los suicidios de personas gays y lesbianas podrían dar cuenta de 30\% del total de suicidios de Estados Unidos de Norteamérica.

En relación a estos índices, se plantea la existencia de factores de riesgos específicos muy significativos como la victimización de género (e.g. Birjett et al., 2009), la homofobia internalizada (e.g. González et al., 2012), la marginación de la comunidad (e.g. O'Donnell et al., 2011) y la edad de "salida del closet" (Mustanski et al., 2013).

\section{Principales resultados de las investigaciones}

Los estudios epidemiológicos muestran resultados que agregan evidencia a lo planteado por la 
literatura previa en relación a los malos indicadores de salud mental y a la alta incidencia y prevalencia de comportamientos suicidas (ideación e intentos) en poblaciones LGBT. Específicamente, se replican los hallazgos previos que señalan que el riesgo suicida es mucho mayor en estas poblaciones al compararlas con población heterosexual y que las poblaciones LGBT presentan una mayor probabilidad de intentos suicidas.

Un poco más de la mitad de los estudios analizados aportan con evidencia al modelo de estrés de minorías, planteando en términos generales que identificarse como LGBT constituye un predictor de tendencias suicidas (e.g. Walls et al., 2008). Junto con lo anterior, una mayoría de las investigaciones revisadas constatan la relación entre victimización de la población LGBT y comportamientos suicidas. Específicamente, el abuso físico, psicológico y sexual, al que se ven expuestas las personas LGBT debido a su identidad de género y orientación sexual, fue asociado con suicidalidad (e.g. Corliss et al., 2010). Asimismo, se observa que la falta de apoyo social tiene efectos nocivos en la salud mental de las personas LGBT, efectos que constituyen en sí mismos factores de riesgo (e.g. Nemoto et al., 2011; Walls et al., 2008). Además, algunas de las investigaciones analizadas presentan evidencia respecto al rol mediador de la aceptación o rechazo familiar de la identidad de género y orientación sexual respecto del riesgo suicida (e.g., Diamond, 2013; Bockting et al., 2013), coincidiendo con el planteamiento de que adolescentes LGB que experimentan rechazo familiar serían ocho veces más propensos a intentar suicidarse (Blosnich, 2012). Un estudio destaca la relación entre homofobia internalizada y tendencias suicidas en personas gay, señalando que el comportamiento suicida sería una manera de castigar o destruir la "parte mala" de sí mismo (McAndrew, 2010). En relación con el proceso de adopción de la identidad de género y orientación sexual, los hallazgos de un estudio (Birkett et al., 2009) muestran que la etapa de "cuestionamiento" constituye un importante período de riesgo suicida en el caso de las personas LGBT. En relación con la edad de "salida del closet", se encontró que a menor edad de develación de la identidad de género y orientación sexual LGBT, mayor probabilidad de victimización de género y mayor riesgo de comportamiento suicida (Corliss et al., 2010).

Con respecto a los factores protectores y de resiliencia de la suicidalidad, la presencia de apoyo social en general fue el más frecuentemente reportado. Específicamente, se muestra la importancia del apoyo familiar (Mustanski et al., 2013; Espelage et al., 2008), del apoyo de grupo de pares (Moody et al., 2013) y la presencia de un ambiente social inclusivo (Espelage et al., 2008; McAndrew, 2010) como factores protectores.

\section{Discusión}

Esta revisión sistemática muestra que la investigación sobre suicidabilidad, concentrada en la ideación y los intentos de suicidio en personas LGBT, ha aumentado durante la última década. La gran mayoría de las investigaciones han sido realizadas en Estados Unidos de Norteamérica y otros países de habla inglesa. A su vez, estos estudios han sido publicados en importantes revistas especializadas principalmente en salud mental y salud pública y referidas a minorías sexuales. En conjunto, proveen un conocimiento significativo sobre los factores protectores y de riesgo suicida, así como de su epidemiología para esta población en particular.

Algunos de los hallazgos más importantes muestran que en la población LGBT los procesos relacionados con el suicidio (ideación e intentos) son más prevalentes que en la población heterosexual. Comprender cuáles son las condiciones y factores que inciden en este proceso de salud mental en dicha población es muy relevante dado el nivel de vulnerabilidad de las personas LGBT evidenciada en los estudios y la relación de esta con la construcción sociocultural de la diversidad sexual asociada al estigma, discriminación, distintas formas de violencia, faltas de apoyo, barreras de uso de los servicios de salud, entre otros fenómenos. $\mathrm{Al}$ respecto, interesante es notar que la mayoría de los países en que fueron realizadas las investigaciones revisadas han impulsado importantes políticas de inclusión que, sin embargo, dado los resultados de dichos estudios, no parecen condecirse con una disminución de la incidencia de problemas de salud mental y suicidabilidad asociados a la victimización de género.

La revisión de las investigaciones también muestra que estas presentan limitaciones importantes que debiesen ser abordadas en futuros estudios. Así, por ejemplo, la mayoría de estos han sido 
realizados con muestras de un muy amplio rango de edades. Esto es problemático ya que implica un gran número de variables confundentes relativas a las etapas de desarrollo en general y a las etapas de identificación de género y orientación sexual en particular.

Junto con lo anterior, se observa falta de innovación metodológica en términos de los diseños de investigación. Muy pocos de los estudios examinados emplean metodología cualitativa o son diseñados con el propósito de aumentar la comprensión de las interrelaciones entre los factores de riesgo y factores protectores, la dimensión cultural, la diversidad sexual y el suicidio. En este sentido, Haas et al. (2010) han abogado por un enfoque culturalmente-sensible para el estudio de la suicidabilidad que considere dimensiones subjetivas e idiosincráticas asociadas al origen, al grupo etario, etnicidad, diversidad sexual, entre otros. En este contexto, resulta necesario no sólo el desarrollo de estudios epidemiológicos que nos indiquen la envergadura de este problema en Chile, sino que también la generación de un conocimiento de alcance local que permita desarrollar políticas y programas anclados en las características particulares y complejas que adquiere el fenómeno del comportamiento suicida en personas LGBT que habitan nuestra cultura con sus específicas formas de significación, estigmatización y discriminación de la diversidad sexual y de género.

Por otro lado, sólo un estudio de los revisados se ha centrado en evaluar el efecto de una intervención preventiva, mientras que sólo uno ha sido publicado en una revista especializada en investigación del suicidio. Estos dos ejemplos sugieren que el desarrollo del conocimiento en la investigación sobre el suicidio en población LGBT sigue siendo exploratoria, focalizada mayormente en estudios de carácter epidemiológico y concentrado en países desarrollados. Esto refleja que una buena parte del conocimiento científico en esta área ha seguido el desarrollo de los procesos culturales y sociales que han tenido algunos países. Por ahora, el tema se ha concentrado en el área de las políticas de salud y de los derechos de las minorías sexuales, pero no todavía en un interés académico y disciplinar más específico. Se requiere mayor atención de la comunidad especializada en investigación del suicidio sobre este grupo de riesgo, con el fin de proporcionar conocimiento útil para la prevención y el desarrollo de interven- ciones efectivas y pertinentes, pues comprender las necesidades en salud mental de esta población no es sólo materia de salud pública, sino finalmente de derechos humanos.

\section{Referencias}

1. Organización Mundial de la Salud (OMS). Informe mundial sobre la violencia y la salud. Ginebra: Organización Mundial de la Salud; 2003.

2. Cochran S, Mays V. Lifetime prevalence of suicide symptoms and affective disorders among men reporting same-sex sexual partners: Results from NHANES III. American Journal Of Public Health 2000; 90 (4): 573-8.

3. Goldsmith S, Pellmar T, Kleinman A, Bunney W. Reducing suicide: A national imperative. Washington, DC, US: National Academies Press; 2002.

4. Haas A, Eliason M, Mays V, Mathy R, Cochran S, Clayton $\mathrm{P}$, et al. Suicide and suicide risk in lesbian, gay, bisexual, and transgender populations: Review and recommendations. Journal Of Homosexuality 2011; 58 (1): 10-51.

5. King M, Semlyen J, Tai S, Killaspy H, Osborn D, Nazareth I, et al. A systematic review of mental disorder, suicide, and deliberate self harm in lesbian, gay and bisexual people. BMC Psychiatry 2008; 8: 70.

6. Lewis N. Mental health in sexual minorities: recent indicators, trends and their relationship to place in North America and Europe. Health Place 2009; 15 (4): 102945.

7. Logie C. The Case for the World Health Organization's Commission on the Social Determinants of Health to Address Sexual Orientation. Am J Public Health 2012; 102 (7): 1243-6.

8. Meyer IH. Prejudice, social stress, and mental health in lesbian, gay, and bisexual populations: Conceptual issues and research evidence. Psychol Bull 2003; 129 (5): 674-97.

9. Meyer IH, Dietrich J, Schwartz S. Lifetime Prevalence of Mental Disorders and Suicide Attempts in Diverse Lesbian, Gay, and Bisexual Populations. Am J Public Health 2008; 98 (6): 1004-6.

10. Pineda C. Factores asociados con riesgo de suicidio de adolescentes y jóvenes autoidentificados como lesbianas, gays y bisexuales: Estado actual de la literatura. Rev Colomb Psiquiat 2013; 42 (4): 333-49.

11. Berredo de Toledo, L. Dificultades Administrativas Enfrentadas por las Personas Trans en la Región Metropolitana de Chile. [tesina en Internet]. Santiago: Universidad Academia de Humanismo Cristiano; 2011. 
Disponible en: http://bibliotecadigital.academia.cl/ handle/123456789/2188 [Consultado el 10 de diciembre de 2015].

12. León M, Del Río P, Chaigneau S. Nivel de Sintomatología Psicopatológica en Población Adolescente Femenina de Santiago de Chile, Según Orientación Sexual. Rev Chil Psiquiatr Neurol Infanc Adolesc 2012; 23 (10): 26.

13. Universidad Cayetano Heredia. Estudio a través de internet sobre "Bullying", y sus manifestaciones homofóbicas en escuelas de Chile, Guatemala, México y Perú, y su impacto en la salud de jóvenes varones entre 18 y 24 años. Instituto de Estudios en Salud, Sexualidad y Desarrollo Humano. Lima; 2011. Disponible en: http://www. iessdeh.org/index.php/publicaciones-1/informes-db [Consultado el 10 de diciembre de 2015].

14. Peña F, Rodríguez J. Orientaciones generales de atención a niños, niñas y adolescentes lesbianas, gays, bisexuales y trans para profesionales de la salud mental. Santiago: Fundación Todo Mejora; 2013. Disponible en: http:// todomejora.org/guias/ [Consultado el 10 de diciembre de 2015].

15. Liberati A, Altman DG, Tetzlaff J, Mulrow C, Gøtzsche PC, Ioannidis JPA, et al. The PRISMA statement for reporting systematic reviews and meta-analyses of studies that evaluate health care interventions: explanation and elaboration. J Clin Epidemiol 2009; 62 (10): e1-34. 